\section{Wie ich lebe und warum}

Die Fotofolge »Wie ich lebe und warum « wurde in zwei verschiedenen Versionen publiziert: $1970 \mathrm{im}$ Sammelband Trivialmythen, herausgegeben von Renate Matthaei im März-Verlag (hier: [67]-[73]), und in einer "Neufassung 1974« (FW, 310) mit leichten Veränderungen (vgl. FW, 143-[149]) in der von Maleen Brinkmann posthum vorgenommenen Zusammenstellung Der Film in Worten (1982). Beide Bilderfolgen umfassen sechs Seiten, welche aus je zwei neben- und drei untereinander montierten SchwarzWeiß-Fotografien bestehen; eine Struktur, die sich in den Fotosequenzen des Gedichtbands Westwärts $1 \& 2$ wiederholt (vgl. Ww, vor 1 und nach 335). In »Wie ich lebe und warum « ist jeweils Brinkmanns Lebensraum in Köln zu sehen, seine Wohnung und deren unmittelbare Umgebung. In der überarbeiteten Version sind einzelne Bilder auf einer anderen Position und mehr Außenaufnahmen eingefügt; es fehlen die Darstellungen von Brinkmanns Frau und Sohn in der Wohnung; $\mathrm{zu}$ sehen sind jetzt (vermutlich Brinkmanns) Beine und Schuhe.

Die Bilder stehen durch Auswahl und Inszenierung der Objekte (s. Kap. 12) und durch die Wiederholung einzelner Motive (wie beispielsweise ein Dachfenster) in enger Verbindung. Auch ist in beiden Versionen das erste Bild die leicht geöffnete Wohnungstür, während das letzte die geschlossene Haustür zeigt. In beiden steckt der Schlüsselbund, so dass der Eindruck entsteht, man werde von Brinkmann in seinen Mikrokosmos mit hineingenommen und am Ende wieder hinausbegleitet. Dazwischen finden sich Aufnahmen eher ungewöhnlicher Ausschnitte seiner Wohnung und Nachbarschaft: Müll und Schrott, sanitäre Anlagen, kaputte Rollläden, Berge von Altpapier, der Blick auf dunkle Hinterhöfe oder Straßenfluchten. Die symmetrische Setzung der Bilder in Verbindung mit der Rahmung durch die Aufnahmen der Türen, legt eine Leserichtung nahe und provoziert so eine textverwandte Rezeption, welche Steinaecker folgendermaßen beschreibt: »Das einzige - in beiden Fassungen insgesamt fünfmal und damit fast auf jeder Seite wiederkehrende Motiv, die Ansicht von Treppenstufen, fungiert dabei semantisch als Scharnierstück zwischen den wechselnden Schauplätzen und semiotisch als Satzzeichen. Erfolgt doch die Anordnung der Fotos hier, anders als in den Collagebüchern, syntagmatisch: Die 'Serie ' wird zum Text, der eben nicht nur das ,Wie der Lebensweise zu zeigen versucht, sondern auch [...] das ।Warum « (Steinaecker 2007, 165 f.).
Das titelgebende »Wie ich lebe« bezieht sich dabei ebenso auf die Tatsache der Dokumentation des Lebensraums, als auch auf die Art der Darstellung und Präsentation dessen, während das "Warum« über das vermeintlich Faktische, Sichtbare hinaus, Bezug nimmt $\mathrm{zu}$ anderen Kategorien der (existentiellen) Bedeutungszuschreibung. Damit ist auch Brinkmanns Selbstverständnis als Künstler und Autor visualisiert. Die Bildfolge ist durch ihre Benennung und Gestaltung intermedial ausgerichtet und nimmt Bezug zu sprachlichen Sinngebungsverfahren, während die Abbildungen ihre Stärke bewahren. Ihre Erscheinung als reine Oberfläche schafft einen Freiraum der Deutung. Ähnlich äußert sich die Erstherausgeberin über Brinkmanns Beitrag in ihrem Vorwort: "Brinkmann zeigt des Private als triviale Fiktion unmittelbar: in einer Serie von Fotos aus seinem >Millieu . Literatur reflektiert er hier in einem anderen Medium als das, was sie tatsächlich für ihn ist: als `Bild`, das die Kamera seiner Sensibilität der Umwelt entnimmt, verändert und reproduziert [...]. Insgesamt ist das, was vorliegt, ein Konzentrat der Oberfläche, die, gerade weil sie sich als Oberfläche versteht, auch mehr ist: Poesie und Polemik» (Matthaei 1970, 8-10).

Brinkmanns Fotoserie ist auch eine visuelle Spur seiner Poetologie (s. Kap. 15): Sie ist eine Dokumentation des radikal subjektiven Blicks in der möglichst konkreten, künstlerischen Darstellung, welche in der Lage ist, »eine Tür aufzumachen, aus der Sprache und den Festlegungen raus " (Ww, 9). Durch die dabei entstehende Bewegung in der Abfolge von Bildern, rückt diese Arbeit zudem in die Nähe intermedialer Auseinandersetzungen Brinkmanns mit dem Filmischen (s. Kap. 13, 44). Somit kann die Fotosequenz auch als eine Visualisierung seines Diktums verstanden werden: »denn das Leben ist ein komplexer Bildzusammenhang. Es kommt darauf an, in welchen Bildern wir leben und mit welchen Bildern wir unsere eigenen Bilder koppeln« (Silverscreen, 8).

\section{Literatur}

Brinkmann, Maleen: Editorische Notiz. In: Dies.: (Hg.) Der Film in Worten. Reinbek bei Hamburg 1982.

Matthaei, Renate (Hg.): Trivialmythen. Frankfurt a. M. 1970.

Matthaei, Renate: Vorwort. In: Dies. (Hg.): Trivialmythen.

Frankfurt a. M. 1970, 7-10.

von Steinaecker, Thomas: Literarische Foto-Texte: zur Funktion der Fotografien in den Texten Rolf Dieter Brinkmanns, Alexander Kluges und W. G. Sebalds. Bielefeld 2007.

Stephanie Schmitt 